\title{
Induction of labour at 41 weeks versus expectant management and induction of labour at 42 weeks (SWEdish Post-term Induction Study, SWEPIS): multicentre, open label, randomised, superiority trial
}

\author{
Ulla-Britt Wennerholm, ${ }^{1}$ Sissel Saltvedt, ${ }^{2}$ Anna Wessberg, ${ }^{3}$ Mårten Alkmark, ${ }^{1}$ Christina Bergh, ${ }^{1}$ \\ Sophia Brismar Wendel, ${ }^{4}$ Helena Fadl, ${ }^{5}$ Maria Jonsson, ${ }^{6}$ Lars Ladfors, ${ }^{1}$ Verena Sengpiel, ${ }^{1}$ \\ Jan Wesström, ${ }^{7}$ Göran Wennergren, ${ }^{8}$ Anna-Karin Wikström, ${ }^{6}$ Helen Elden, ${ }^{3}$ Olof Stephansson, ${ }^{9}$ \\ Henrik Hagberg ${ }^{1}$
}

For numbered affiliations see end of the article.

Correspondence to:

U-B Wennerholm ulla-britt. wennerholm@vgregion.se (ORCID 0000-0003-2475-2226) Additional material is published online only. To view please visit the journal online.

Cite this as: BMJ 2019;367:16131 http://dx.doi.org/10.1136/bmj.16131 Accepted: 16 October 2019

\begin{abstract}
OBJECTIVE

To evaluate if induction of labour at 41 weeks improves perinatal and maternal outcomes in women with a low risk pregnancy compared with expectant management and induction of labour at 42 weeks.

DESIGN

Multicentre, open label, randomised controlled superiority trial.

SETTING

14 hospitals in Sweden, 2016-18.

PARTICIPANTS

2760 women with a low risk uncomplicated singleton pregnancy randomised $(1: 1)$ by the Swedish Pregnancy Register. 1381 women were assigned to the induction group and 1379 were assigned to the expectant management group.

INTERVENTIONS

Induction of labour at 41 weeks and expectant management and induction of labour at 42 weeks.

MAIN OUTCOME MEASURES

The primary outcome was a composite perinatal outcome including one or more of stillbirth, neonatal mortality, Apgar score less than 7 at five minutes, $\mathrm{pH}$ less than 7.00 or metabolic acidosis ( $\mathrm{pH}<7.05$ and base deficit $>12 \mathrm{mmol} / \mathrm{L}$ ) in the umbilical artery, hypoxic ischaemic encephalopathy, intracranial haemorrhage, convulsions, meconium aspiration syndrome, mechanical ventilation within 72 hours, or
\end{abstract}

obstetric brachial plexus injury. Primary analysis was by intention to treat.

\section{RESULTS}

The study was stopped early owing to a significantly higher rate of perinatal mortality in the expectant management group. The composite primary perinatal outcome did not differ between the groups: $2.4 \%$

(33/1381) in the induction group and $2.2 \%$

$(31 / 1379)$ in the expectant management group (relative risk 1.06, 95\% confidence interval 0.65 to $1.73 ; \mathrm{P}=0.90)$. No perinatal deaths occurred in the induction group but six (five stillbirths and one early neonatal death) occurred in the expectant management group $(P=0.03)$. The proportion of caesarean delivery, instrumental vaginal delivery, or any major maternal morbidity did not differ between the groups.

\section{CONCLUSIONS}

This study comparing induction of labour at 41 weeks with expectant management and induction at 42 weeks does not show any significant difference in the primary composite adverse perinatal outcome. However, a reduction of the secondary outcome perinatal mortality is observed without increasing adverse maternal outcomes. Although these results should be interpreted cautiously, induction of labour ought to be offered to women no later than at 41 weeks and could be one (of few) interventions that reduces the rate of stillbirths.

TRIAL REGISTRATION

Current Controlled Trials ISRCTN26113652.

\section{Introduction}

Adverse perinatal outcomes gradually increase after 40 gestational weeks and are substantially increased post-term ( $\geq 42$ weeks ( $\geq 294$ days)). ${ }^{12}$ The risk of stillbirth has been shown to increase after term, ${ }^{1-5}$ and worldwide as much as $14 \%$ of stillbirths are associated with prolonged pregnancy. ${ }^{2}$ Furthermore, maternal complications also increase with duration of pregnancy after 40 weeks. ${ }^{1}$ To date, no agreement exists on how to manage late term ( 41 weeks +0 days to 42 weeks +0 days) pregnancies. The World Health Organization recommends induction of labour at 41 weeks, $^{6}$ and many countries offer induction of labour between 41 and 42 weeks to avoid prolonged pregnancy. $^{78}$ 
Randomised controlled trials have compared induction of labour with expectant management in prolonged pregnancies, most with inconclusive results for perinatal mortality and major morbidity. ${ }^{9}$ The results from the latest Cochrane review (2018) showed lower rates of caesarean delivery and perinatal death but a higher rate of operative vaginal delivery in the induction group compared with the expectant management group. ${ }^{9}$ After the latest Cochrane review and after the initiation of the present study, ${ }^{10}$ two large randomised controlled trials examining low risk pregnancies have been published. A large trial from the United States, ARRIVE (A Randomized Trial of Induction Versus Expectant Management), compared induction of labour in nulliparous women at 39 weeks +0 days to 39 weeks +4 days with expectant management until 41 weeks+0 days. ${ }^{11}$ No significant difference was found in perinatal outcome between groups, whereas the frequency of caesarean delivery was significantly lower in the early induction group. Another large recent trial from the Netherlands, INDEX (INDuction of labour at 41 weeks with a policy of EXpectant management until 42 weeks), compared induction of labour at 41 weeks +0 days to 41 weeks +1 day with expectant management until 42 weeks +0 days. ${ }^{12}$ The results could not confirm non-inferiority for adverse perinatal outcome of expectant management, instead a significantly higher risk of adverse perinatal outcome was found in the expectant management group. No significant difference in the rate of caesarean delivery was found.

The current practice in many centres in the United Kingdom and Scandinavia is to induce delivery no later than 42 weeks, but several studies suggest that the risk of perinatal mortality and morbidity has actually already increased significantly at 41 weeks. ${ }^{3-5}$ The risk of stillbirth increases gradually from 39 weeks of gestation ${ }^{13}$ and increases exponentially as the pregnancy approaches 42 weeks, ${ }^{3-5} 13$ whereas the risk of neonatal mortality is not increased until 42 weeks according to most studies. ${ }^{3-513}$ We therefore found it clinically justified to compare induction of labour at 41 weeks with expectant management and induction at 42 weeks for maternal and perinatal outcomes. At the start of the present trial, only two studies (one was an abstract) out of 30 included in the Cochrane review specifically compared induction of labour at 41 weeks with expectant management until 42 weeks. ${ }^{1415}$

We evaluated if induction of labour at 41 weeks $+0-2$ days compared with expectant management and induction of labour at 42 weeks $+0-1$ days was superior in terms of perinatal outcome in healthy women with a low risk pregnancy.

\section{Methods}

Study design

SWEPIS (SWEdish Post-term Induction Study) was a multicentre, open label, randomised controlled superiority trial conducted in Sweden from May 2016 to October 2018. The trial was register based, with randomisation and most data collection done by using the Swedish Pregnancy Register. ${ }^{16}$ Fourteen hospitals with antenatal clinics linked to the register were involved in the trial. Five of the hospitals were university clinics and nine were county hospitals comprising about 60000 deliveries per year of the around 115000 to 120000 annual deliveries in Sweden. The trial was conducted according to the CONSORT guidelines. The protocol is available online (www-ncbi-nlm-nih-gov. proxy.kib.ki.se/pubmed/26951777) and as a publication. ${ }^{10}$ The trial was undertaken within the Swedish Network for National Clinical Studies within Obstetrics and Gynaecology (SNAKS).

\section{Participants}

Pregnant women were eligible for participation if they were aged 18 or more, understood oral and written information, and had a singleton pregnancy with a fetus in cephalic presentation at 40 weeks +6 days to 41 weeks+1 day according to ultrasound based dating in the first or early second trimester or for pregnancies after assisted reproduction according to the day of oocyte retrieval. Exclusion criteria were previous caesarean delivery or other uterine surgery, pregestational and insulin dependent gestational diabetes, hypertensive disorder of pregnancy, known oligohydramnios (amniotic fluid index $<50 \mathrm{~mm}$ or deepest vertical pocket $<20 \mathrm{~mm}$ ) or small for gestational age fetus (estimated fetal weight $\leq 2$ standard deviations according to the sex and gestational age specific Swedish reference), ${ }^{17}$ diagnosed fetal malformation, contraindication to vaginal delivery, and any other maternal condition affecting the progress of the pregnancy to 42 weeks.

\section{Study logistics}

General information about the study was provided in the form of posters or videos in the waiting rooms at the antenatal clinics and by advertising in local newspapers. More detailed information was provided on the study website. When the pregnancies were at around 40 weeks, the midwives provided women with an oral account of the study in Swedish or written information in any of 17 other languages applicable to women who were non-Swedish. In the Stockholm region (five clinics), women were enrolled in association with a 41 week ultrasound scan, which is offered to all pregnant women in the region. This is a voluntary procedure, with almost $100 \%$ coverage, aiming to confirm a normal pregnancy (defined as mean fetal abdominal diameter $>110 \mathrm{~mm}$ and normal amniotic fluid) before proceeding to 42 weeks. The midwife performing the ultrasonography answered questions about the study and handled the randomisation after written informed consent was obtained. In all other centres, women interested in taking part were invited to visit a research midwife who managed patient consent and randomisation. Outside the Stockholm region, 41 week scans were not routinely offered.

\section{Randomisation and masking}

Randomisation was done between 40 weeks +6 days and 41 weeks +1 day. Enrolled women were allocated 
to the induction group or expectant management group (controls). In the induction group, labour was induced within 24 hours of randomisation (ie, same or next day) but not earlier than 41 weeks +0 days. In the expectant management group, labour was induced at 42 weeks +0 days to 42 weeks +1 day.

Allocation to a trial group, 1:1, was done with central online randomisation by dynamic allocation, a method that actively minimises the imbalance between the groups for each new patient that is randomised. Centre and parity (primiparity versus multiparity) were used as minimisation variables.

The Swedish Pregnancy Register ${ }^{16}$ set up the randomisation module, which was incorporated in the register but separate from the register data. Access to the randomisation module used a separate log-in system. The module also included an electronic case report form. After delivery and the neonatal period, we used the women's unique personal identification number to retrieve data on antenatal, delivery, and neonatal characteristics from the Swedish Pregnancy Register and Swedish Neonatal Quality Register. ${ }^{18}$ Because most variables in the study were included in the quality registers, the study could be performed relatively fast and at low cost.

Owing to the nature of the intervention it was not possible to blind participants or care givers.

\section{Strategies}

Induction of labour was carried out in the same way in both groups. At admission, the women were examined for blood pressure, proteinuria, fetal presentation by abdominal palpation, cervical status, and fetal wellbeing by cardiotocography. Amniotomy was performed if the fetal head was well engaged and the cervix was ripe (Bishop score $\geq 6$ for primiparous women and $\geq 5$ for multiparous women), followed by oxytocin infusion after 1-2 hours without spontaneous regular contractions. If the fetal head was not engaged or the cervix was less ripe, any of the following methods was used according to local routines: mechanical dilation with a Foley-like catheter, prostaglandin E1 (misoprostol, oral or vaginal), or prostaglandin E2 (dinoprostone, vaginal).

After randomisation, no monitoring was offered within the framework of the trial. In Sweden, most antenatal clinics offer one follow-up visit after term, usually around 41 weeks, including measurement of blood pressure, fundal height, and fetal heart rate by doptone. Further examinations, induction of labour, or caesarean delivery are initiated for usual obstetric indications, such as decreased fetal movements, suspected fetal growth restriction, or pre-eclampsia. After 41 weeks, the threshold for interventions is low. Indication for a scheduled caesarean section included undiagnosed breech or transverse presentation with failed external version. Fetal scalp blood sampling $(\mathrm{pH}$ or lactate) was performed during labour when indicated.

\section{Outcomes}

The primary outcome was a composite perinatal outcome of mortality and morbidity. Perinatal mortality was defined as stillbirth and neonatal death (days 0-27). Neonatal morbidity was defined as one or more of several outcomes: Apgar score less than 7 at five minutes, $\mathrm{pH}$ less than 7.00 or metabolic acidosis $(\mathrm{pH}$ $<7.05$ and base deficit $>12 \mathrm{mmol} / \mathrm{L}$ ) in the umbilical artery, hypoxic ischaemic encephalopathy grades $1-3$, intracranial haemorrhage, convulsions, meconium aspiration syndrome, mechanical ventilation within 72 hours, or obstetric brachial plexus injury.

Secondary neonatal outcomes were the individual components of the primary perinatal outcome, admission to a neonatal intensive care unit, Apgar score less than 4 at five minutes, birth weight, macrosomia ( $\geq 4500 \mathrm{~g}$ ), neonatal jaundice, therapeutic hypothermia, pneumonia, or sepsis.

Secondary maternal outcomes were use of epidural anaesthesia, caesarean delivery, operative vaginal delivery, duration of labour (from onset of regular contractions to delivery), chorioamnionitis, shoulder dystocia, third or fourth degree perineal tear, postpartum haemorrhage $(>1000 \mathrm{~mL})$, wound infection, urinary tract infection, endometritis, sepsis, and breastfeeding at discharge from hospital and at four weeks post partum.

Exploratory neonatal outcomes were neonatal hypoglycaemia, birth trauma (fracture of long bone, clavicle, or skull, other neurological injury, retinal haemorrhage, or facial nerve palsy), small for gestational age, ${ }^{17}$ and large for gestational age.${ }^{17}$ Exploratory maternal outcomes were cervical tear, uterine rupture, hypertensive disorders of pregnancy (pre-eclampsia, gestational hypertension, eclampsia), venous thromboembolism, duration of stay in hospital, admission to intensive care unit, and mortality within 42 days.

\section{Data collection}

We retrieved data on maternal background, pregnancy and delivery characteristics, and perinatal outcomes from the Swedish Pregnancy Register and the Swedish Neonatal Quality Register. ${ }^{16}{ }^{18}$ Both are certified national quality registers initiated by Swedish healthcare professionals. Data prospectively entered in standardised electronic medical records by midwives and clinicians during pregnancy, delivery, and post partum is forwarded to the Swedish Pregnancy Register from all antenatal clinics and most delivery clinics. In the same way, the Swedish Neonatal Quality Register collects data on all newborns admitted to neonatal intensive care units at birth or within 28 days of life. We obtained vital statistics on maternal and neonatal mortality from Statistics Sweden.

Study data were linked with data from the Swedish Pregnancy Register, Swedish Neonatal Quality Register, and Statistics Sweden using the unique personal identification number allocated to each person in Sweden at birth or after immigration. ${ }^{19}$

For all newborns with a primary outcome we collected and scrutinised the medical records. The same process was undertaken in the women with a diagnosis of endometritis to rule out misclassification of sepsis. 
To estimate selection bias we compared the baseline characteristics and pregnancy outcomes of our study population with those of the Swedish background population.

\section{Monitoring}

Before the trial started, an independent Data and Safety Monitoring Board comprising a statistician, senior obstetrician, and senior midwife was formed to supervise the trial through regular reviews. The principle investigators reported serious adverse events immediately to the Data and Safety Monitoring Board, defined as any of perinatal or maternal death; need for neonatal intensive care because of meconium aspiration syndrome, asphyxia, intracranial haemorrhage, or other severe condition; severe maternal morbidity with admission to intensive care unit; and complication associated with induction of labour, such as placental abruption at insertion of Foley catheter, or uterine rupture.

An interim analysis was planned when $50 \%$ of the women had been recruited and had delivered.

\section{Sample size and statistical analyses}

To reduce the primary outcome by one third, from $2.7 \%$ to $1.8 \%$ (superiority testing, level of significance 0.05 , power $80 \%$ ) by induction of labour at 41 weeks compared with expectant management until induction at 42 weeks, we needed a sample size of 10038 women, 5019 in each randomisation group. This calculation assumed that for $10 \%$ of the women, management would not be consistent with the assigned strategy, thus also covering the same power for the per protocol analysis as for the intention to treat analysis. The composite primary outcome of $2.7 \%$ was based on data on perinatal outcomes included in our primary outcome in one Swedish region (Region Skåne) between 2000 and 2010.

The statistical analyses were carried out according to a prespecified analysis plan. Main analyses were performed on the intention to treat population. The primary statistical analysis was the comparison between the induction group and the expectant management group for the primary perinatal composite outcome, with Fisher's exact test (lowest one sided P value multiplied by 2) at a significance level of 0.05 . To compare secondary outcomes, we used Fisher's exact test for dichotomous variables, Fisher's nonparametric permutation test for continuous variables, Mantel Haenszel $\chi^{2}$ test for ordered categorical variables, and Pearsons's $\chi^{2}$ test for non-ordered categorical variables. For the primary efficacy variable (the perinatal composite outcome) and dichotomous secondary variables we calculated relative risks with corresponding 95\% confidence intervals between the groups. For continuous secondary variables we calculated mean differences with 95\% confidence intervals between the groups. Data are presented as means with standard deviations, medians with interquartile ranges, and numbers with percentages, as appropriate.
The intention to treat population included all randomised women except those who withdrew consent or were lost to follow-up. In the intention to treat group we included women with spontaneous labour or prelabour rupture of membranes after randomisation but before induction, or with pregnancy complications necessitating interventions for medical reasons.

A post hoc sensitivity analysis for the primary efficacy analysis was performed adjusted for the minimisation variables centre and primiparity or multiparity using multivariable logistic regression analysis with centre as fixed effect.

Complementary analyses were performed for comparison of the primary perinatal composite outcome and secondary efficacy outcomes on the per protocol population. This population comprised all randomised women who completed the study without important deviations from the protocol. We defined the criteria for protocol deviation before data were analysed. For the induction group, protocol deviation was defined as induction at less than 41 weeks+0 days; labour induction, spontaneous labour, or caesarean delivery at more than 41 weeks +2 days because of scheduling error or delivery room unavailability; patient or provider preference; and non-medically indicated elective caesarean delivery. For the expectant management group, protocol deviation was defined as induction at more than 42 weeks +1 day, induction of labour at less than 42 weeks owing to scheduling error or patient or provider preference, and non-medically indicated elective caesarean delivery.

Prespecified subgroup variables were maternal age ( $\geq 35$ years), nulliparity, and body mass index $(\geq 30)$. Logistic regression with treatment subgroup variable and the interaction term treatment $\times$ subgroup variable was used to test whether the effect of treatment differed between subgroups.

All significance tests were two sided at the 0.05 level. Statistical analyses were performed with SAS System Version 9 for Windows (SAS, Cary, NC).

\section{Patient and public involvement}

Pregnant women were not involved in the design, outcome measures, or recruiting plans of the study, and they were not asked to give advice on interpretation of results. The results of the research will be disseminated to the participants and public through broadcasts, popular science articles, and newspapers.

\section{Results}

On 2 October 2018 the Data and Safety Monitoring Board strongly recommended the SWEPIS steering committee to stop the study owing to a statistically significant higher perinatal mortality in the expectant management group. Although perinatal mortality was a secondary outcome, it was not considered ethical to continue the study. No perinatal deaths occurred in the early induction group but six occurred in the expectant management group (five stillbirths and one early neonatal death; $\mathrm{P}=0.03)$. 
Recruitment took place from 20 May 2016 to 13 October 2018. Oral and written informed consent was obtained from 2762 women, who underwent randomisation. Overall, 1383 women were assigned to induction at 41 weeks and 1379 were assigned to expectant management until induction at 42 weeks (fig 1). Supplementary table A shows recruitment according to trial centre. After randomisation but before intervention, two women in the induction group withdrew their consent to participate and for their data to be used, thus 1381 women in the induction group and 1379 women in the expectant management group were included in the intention to treat analysis. The two groups were similar at baseline (table 1).

Compared with the Swedish background population, women in the study groups had a higher level of education and were more often born in Sweden (see supplementary table B). In the induction group, 14.1\% (195/1381) of the women had spontaneous onset of labour, $85.5 \%$ (1181/1381) underwent induction, of whom $76.6 \%$ (905/1181) had cervical ripening, and 0.4\% (5/1381) had a scheduled caesarean delivery (table 2).
In the expectant management group, $66.7 \%$ (920/1379) of the women had spontaneous onset of labour and 33.1\% (457/1379) were induced, of whom $74.4 \%(340 / 457)$ had cervical ripening and $0.1 \%(2 / 1379)$ had a scheduled caesarean delivery. Management was not consistent with the assigned strategy in 3.5\% (48/1381) of women in the induction group and $2.0 \%(28 / 1379)$ in the expectant management group (fig 1).

Median time from randomisation to delivery was 2 days (interquartile range 1-2 days) in the induction group and 4 (2-7) days in the expectant management group (table 2, fig 2). Median gestational age at delivery was 289 (288-289) days in the induction group and 292 (289-294) days in the expectant management group.

\section{Primary outcome}

The primary outcome occurred in 2.4\% (33/1381) of women in the induction group and 2.2\% (31/1379) of women in the expectant management group (relative risk 1.06, 95\% confidence interval 0.65 to 1.73; $\mathrm{P}=0.90$ ) (table 3).

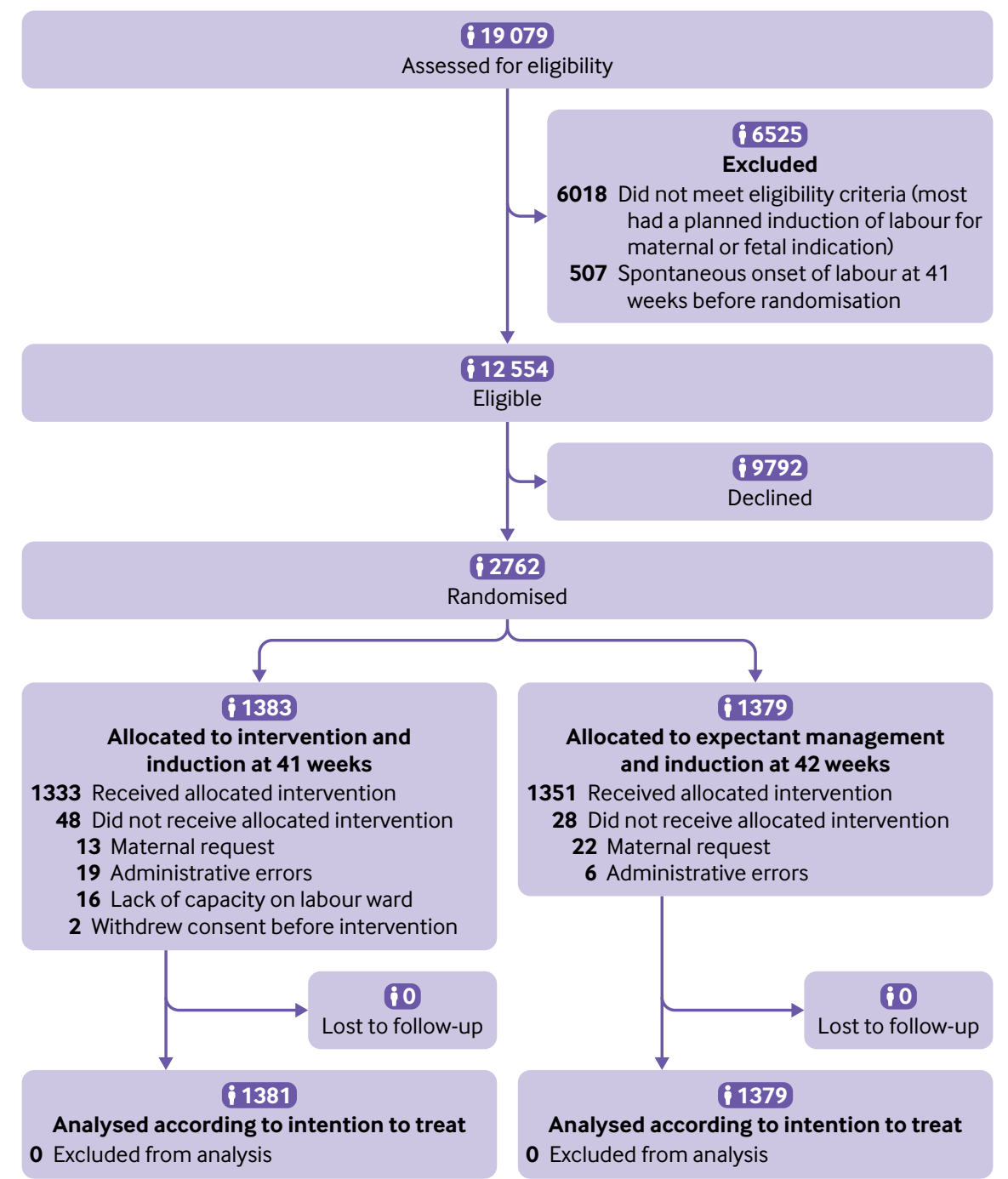

Fig 1 | Flowchart of eligibility, randomisation, delivery, and assessment 


\begin{tabular}{|c|c|c|}
\hline Variables & Induction group $(\mathrm{n}=1381)$ & Expectant management group $(n=1379)$ \\
\hline Age at randomisation (years): & $\mathrm{n}=1381$ & $\mathrm{n}=1379$ \\
\hline Mean (SD) age & $31.2(4.7)$ & $31.1(4.5)$ \\
\hline Median (interquartile range) & $31.1(28.0-34.6)$ & $30.9(27.9-34.2)$ \\
\hline$\$ 35$ years & 1078/1381 (78.1) & $1100 / 1379(79.8)$ \\
\hline$\geq 35$ years & 303/1381 (21.9) & $279 / 1379(20.2)$ \\
\hline \multicolumn{3}{|l|}{ Parity: } \\
\hline Nulliparous & $762 / 1381(55.2)$ & $753 / 1379(54.6)$ \\
\hline Parous & 619/1381 (44.8) & $626 / 1379(45.4)$ \\
\hline \multicolumn{3}{|l|}{ Smoking at first antenatal visit (cigarettes/day): } \\
\hline 0 & $1242 / 1274(97.5)$ & 1228/1276 (96.9) \\
\hline $1-9$ & $25 / 1274(2.0)$ & $30 / 1276(2.4)$ \\
\hline$\geq 10$ & $7 / 1274(0.5)$ & $9 / 1276(0.7)$ \\
\hline \multicolumn{3}{|l|}{ Alcohol screening at first antenatal visit (points)*: } \\
\hline 0-5 (low risk) & 1102/1197 (92.1) & $1109 / 1190(93.2)$ \\
\hline$\geq 6$ (risky behaviour) & 95/1197 (7.9) & $81 / 1190(6.8)$ \\
\hline \multicolumn{3}{|l|}{ Medical history: } \\
\hline Psychiatric disorder & 95/1001 (9.5) & 108/997 (10.8) \\
\hline Prepregnancy diabetes type 1 or 2 & $2 / 1365(0.1)$ & $0 / 1355(0.0)$ \\
\hline Endocrine disease & $83 / 1362(6.1)$ & $97 / 1355(7.2)$ \\
\hline Chronic hypertension & $2 / 1362(0.1)$ & $1 / 1353(0.1)$ \\
\hline Height at first antenatal visit $(\mathrm{cm})$ : & $\mathrm{n}=1275$ & $\mathrm{n}=1273$ \\
\hline Mean (SD) & $167.5(6.2)$ & $167.6(5.9)$ \\
\hline Median (interquartile range) & $167(163-172)$ & $168(163-172)$ \\
\hline Weight at first antenatal visit (kg): & $\mathrm{n}=1247$ & $\mathrm{n}=1241$ \\
\hline Mean (SD) & $70.0(14.3)$ & $70.7(14.5)$ \\
\hline Median (interquartile range) & $67(60-77)$ & $68(60-77)$ \\
\hline BMI at first antenatal visit: & $n=1275$ & $n=1265$ \\
\hline Mean (SD) & $24.9(4.7)$ & $25.1(4.9)$ \\
\hline Median (interquartile range) & $23.9(21.6-27.1)$ & $24.0(21.7-27.4)$ \\
\hline$<30$ & $1118(87.7)$ & $1081(85.5)$ \\
\hline$\geq 30$ & $157(12.3)$ & $184(14.5)$ \\
\hline Last recorded weight during pregnancy (kg): & $\mathrm{n}=1344$ & $n=1336$ \\
\hline Mean (SD) & $83.5(14.4)$ & $84.0(14.6)$ \\
\hline Median (interquartile range) & $82.0(74.0-91.0)$ & $82.0(74.0-92.0)$ \\
\hline \multicolumn{3}{|l|}{ Region of birth: } \\
\hline Sweden & 1069/1289 (82.9) & 1070/1298 (82.4) \\
\hline Other Nordic country & $74 / 1289(5.7)$ & $84 / 1298(6.5)$ \\
\hline Europe outside Nordic countries & $20 / 1289(1.6)$ & $18 / 1298(1.4)$ \\
\hline Outside Europe & $126 / 1289(9.8)$ & $126 / 1298(9.7)$ \\
\hline \multicolumn{3}{|l|}{ Educational level: } \\
\hline ८9 years compulsory school & $7 / 1221(0.6)$ & $10 / 1242(0.8)$ \\
\hline 9 years compulsory school & $47 / 1221(3.8)$ & $47 / 1242(3.8)$ \\
\hline 10-12 years (high school) & $378 / 1221(31.0)$ & $405 / 1242(32.6)$ \\
\hline University or similar & $789 / 1221(64.6)$ & $780 / 1242(62.8)$ \\
\hline \multicolumn{3}{|l|}{ Employment status: } \\
\hline Employed & $1098 / 1296(84.7)$ & $1105 / 1301(84.9)$ \\
\hline Student & $101 / 1296(7.8)$ & $101 / 1301(7.8)$ \\
\hline Maternity leave & 47/1296 (3.6) & $52 / 1301(4.0)$ \\
\hline Unemployed & $17 / 1296(1.3)$ & $18 / 1301(1.4)$ \\
\hline Sick leave & $15 / 1296(1.2)$ & $9 / 1301(0.7)$ \\
\hline Other & $18 / 1296(1.4)$ & $16 / 1301(1.2)$ \\
\hline \multicolumn{3}{|l|}{ Living status: } \\
\hline Cohabitation with partner & $1215 / 1360(89.3)$ & $1211 / 1353(89.5)$ \\
\hline Living alone & $19 / 1360(1.4)$ & $21 / 1352(1.6)$ \\
\hline \multicolumn{3}{|l|}{ Mode of conception: } \\
\hline Assisted (IVF/ICSI) & 67/1381 (4.9) & $53 / 1379(3.8)$ \\
\hline Subfertility & $160 / 1250(12.8)$ & $146 / 1199(12.2)$ \\
\hline
\end{tabular}

No stillbirths or neonatal deaths (0-27 days) occurred in the induction group (mortality rate $0.0 \%$ ), whereas there were five stillbirths and one neonatal death (mortality rate $0.4 \%$ ) in the expectant management group $(\mathrm{P}=0.03)$ between 41 weeks +2 days and 41 weeks +6 days. One stillbirth occurred on the labour ward soon after admittance. The postmortem examination showed a cardiovascular malformation, which according to specialists in paediatric cardiology could not be considered as lethal. In the other four 


\begin{tabular}{|c|c|c|c|c|}
\hline Variables & $\begin{array}{l}\text { Induction group } \\
(n=1381)\end{array}$ & $\begin{array}{l}\text { Expectant management } \\
\text { group }(n=1379)\end{array}$ & $\begin{array}{l}\text { Relative risk } \\
(95 \% \mathrm{Cl})\end{array}$ & $\begin{array}{l}\text { P value; mean }(95 \% \mathrm{Cl}) \\
\text { difference between groups }\end{array}$ \\
\hline Gestational age at delivery (days) & $\mathrm{n}=1381$ & $\mathrm{n}=1379$ & & \\
\hline Mean (SD) & $288.8(1.3)$ & $291.7(2.7)$ & - & $-2.91(-3.07$ to -2.76$)$ \\
\hline Median (interquartile range) & $289(288-289)$ & $292(289-294)$ & - & - \\
\hline Time from randomisation to delivery (days) & $\mathrm{n}=1381$ & $\mathrm{n}=1379$ & & \\
\hline Mean (SD) & $1.76(1.42)$ & $4.66(2.64)$ & - & $-2.91(-3.06$ to -2.75$)$ \\
\hline Median (interquartile range) & $2(1-2)$ & $4(2-7)$ & - & - \\
\hline $\begin{array}{l}\text { Time from admittance to labour } \\
\text { ward to delivery (hours) }\end{array}$ & $\mathrm{n}=1380$ & $\mathrm{n}=1378$ & & \\
\hline Mean (SD) & $20.1(14.8)$ & $13.6(12.2)$ & - & $<0.001 ; 6.49(5.50$ to 7.50$)$ \\
\hline Median (interquartile range) & $16.2(9.2-27.9)$ & $10.4(4.6-19.0)$ & - & - \\
\hline \multicolumn{5}{|l|}{ Onset of birth process } \\
\hline Spontaneous & 195/1381 (14.1) & $920 / 1379(66.7)$ & - & - \\
\hline Scheduled caesarean delivery & $5 / 1381(0.4)$ & $2 / 1379(0.1)$ & - & - \\
\hline Induction: & $1181 / 1381(85.5)$ & $457 / 1379(33.1)$ & - & - \\
\hline Mode of induction: & & & & - \\
\hline Cervical ripening & $905 / 1181(76.6)$ & $340 / 457(74.4)$ & - & - \\
\hline Amniotomy without oxytocin & 130/1181 (11.0) & $45 / 457(9.8)$ & - & - \\
\hline Amniotomy with oxytocin & $146 / 1181(12.4)$ & $72 / 457(15.8)$ & - & 0.18 \\
\hline \multicolumn{5}{|l|}{ Cervical ripening: } \\
\hline First method mechanical & $343 / 905(37.9)$ & $126 / 340(37.1)$ & & - \\
\hline First method drug & $562 / 905(62.1)$ & $214 / 340(62.9)$ & $0.99(0.90$ to 1.09$)$ & 0.84 \\
\hline \multicolumn{5}{|l|}{ Indication for induction } \\
\hline $\begin{array}{l}\text { Randomisation to } 41 \text { weeks and } \\
\text { induction according to protocol }\end{array}$ & $1146 / 1181(97.0)$ & $0(0.0)$ & - & - \\
\hline $\begin{array}{l}\text { Randomisation to } 42 \text { weeks and } \\
\text { induction according to protocol }\end{array}$ & $0 / 1181(0.0)$ & $373 / 457(81.6)$ & - & - \\
\hline Maternal condition & $1 / 1181(0.1)$ & $38 / 457(8.3)$ & - & - \\
\hline Fetal condition & $0(0.0)$ & $18 / 457(3.9)$ & - & - \\
\hline Maternal request* & $6 / 1181(0.5)$ & $23 / 457(5.0)$ & - & - \\
\hline Othert & $28 / 1181(2.4)$ & $5 / 457(1.1)$ & - & - \\
\hline Duration of labour (hours) & $\mathrm{n}=717$ & $\mathrm{n}=880$ & & \\
\hline Mean (SD) & $7.13(5.39)$ & $8.32(5.94)$ & - & $<0.001,1.19(-1.76$ to -0.64$)$ \\
\hline Median (interquartile range) & $5.67(2.85-10.28)$ & $6.86(3.76-11.45)$ & - & - \\
\hline Use of epidural anaesthesia & $729 / 1381(52.8)$ & $669 / 1379(48.5)$ & 1.09 (1.01 to 1.17$)$ & 0.03 \\
\hline Meconium stained amniotic fluid & $233 / 1238(18.8)$ & $320 / 1127(28.4)$ & $0.66(0.57$ to 0.77$)$ & $<0.001$ \\
\hline \multicolumn{5}{|l|}{ Mode of delivery } \\
\hline Non-instrumental vaginal & $1150 / 1381(83.3)$ & $1140 / 1379(82.7)$ & $1.01(0.97$ to 1.04$)$ & 0.71 \\
\hline Caesarean section & $143 / 1381(10.4)$ & $148 / 1379(10.7)$ & $0.96(0.78$ to 1.20$)$ & 0.79 \\
\hline Assisted vaginal & $88 / 1381(6.4)$ & $91 / 1379(6.6)$ & $0.97(0.73$ to 1.28$)$ & 0.87 \\
\hline Emergency caesarean & $138 / 143(96.5)$ & $146 / 148(98.6)$ & $0.98(0.94$ to 1.01$)$ & 0.42 \\
\hline \multicolumn{5}{|l|}{ Indication for emergency caesarean } \\
\hline Failed induction $\neq$ & $8 / 138(5.8)$ & $7 / 146(4.8)$ & - & - \\
\hline Failure to progress at first stage & $60 / 138(43.5)$ & $53 / 146(36.3)$ & - & - \\
\hline Fetal distress at first stage & $35 / 138(25.4)$ & 28/146 (19.2) & - & - \\
\hline Failure to progress and fetal distress at first stage & $6 / 138(4.3)$ & $7 / 146(4.8)$ & - & - \\
\hline Other indication at first stage & $5 / 138(3.6)$ & $5 / 146(3.4)$ & - & - \\
\hline Failure to progress at second stage & $11 / 138(8.0)$ & $23 / 146(15.8)$ & - & - \\
\hline Fetal distress at second stage & $6 / 138(4.3)$ & $8 / 146(5.5)$ & - & - \\
\hline Failure to progress and fetal & $0(0.0)$ & $3 / 146(2.1)$ & - & - \\
\hline
\end{tabular}

distress at second stage

Failure of operative vaginal delivery

$7 / 138(5.1) \quad 12 / 146(8.2)$

0.27

Indication for assisted vaginal delivery

Failure to progress

$37 / 88(42.0)$

Fetal distress

Failure to progress and fetal distress

Maternal distress

Other

Duration of hospital stay from

delivery to discharge (hours)

Mean (SD)

Median (interquartile range)

Breast feeding

At discharge from delivery hospital

$33 / 88(37.5)$

$5 / 88(5.7)$

$13 / 88(14.8)$

$0(0.0)$

$n=1333$

$28 / 91(30.8)$

$34 / 91(37.4)$

$7 / 91(7.7)$

21/91 (23.1)

1/91 (1.1)

$\mathrm{n}=1333$

4 weeks after delivery

$46.3(27.0)$

$47.1(29.7)$

$43.6(25.3-61.6)$

$44.8(25.8-61.2)$

$987 / 1019(96.9) \quad 981 / 1014(96.7)$

$824 / 939(87.8)$

1.00 (0.99 to 1.02$)$

0.98

835/923 (90.5)

1.03 (1.00 to 1.06$)$

0.35

request before 42 weeks +0 days).

tInduction outside study protocol because of administrative errors or lack of capacity on labour ward.

‡Caesarean section performed when active labour was not reached despite different methods for induction of labour being used, usually for at least 48 hours. 


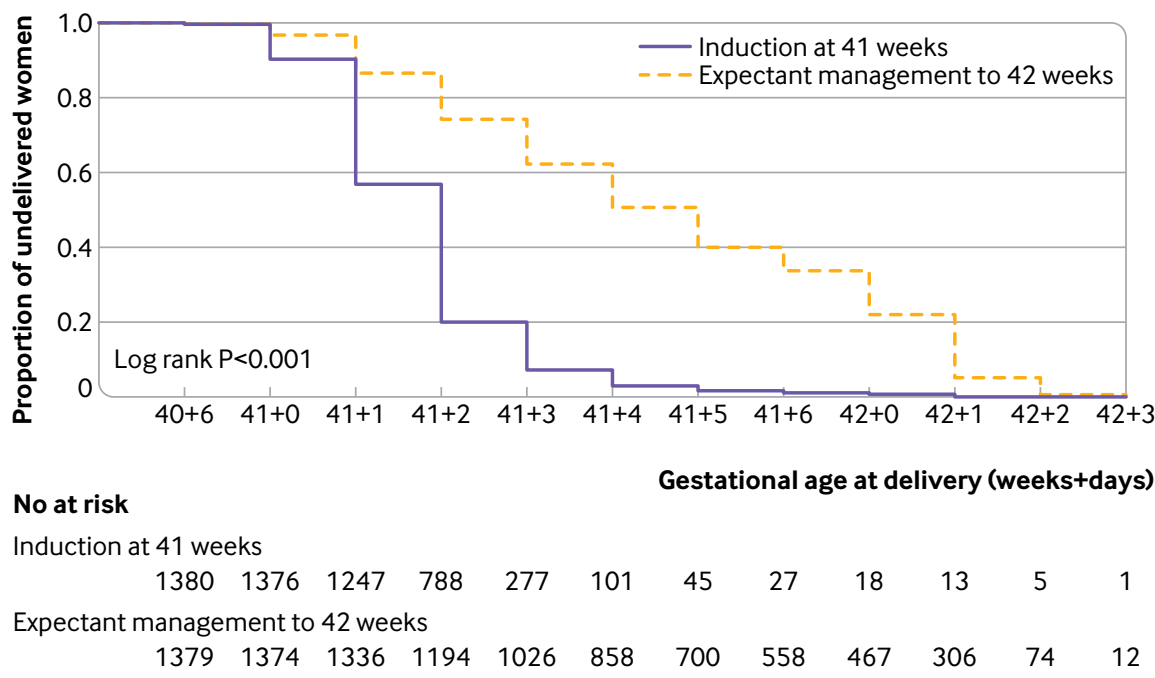

Fig 2 | Gestational age at delivery in intention to treat groups. The induction group included 1380 women because one woman was incorrectly randomised before 40 weeks +6 days and delivered before 40 weeks 6 days

stillbirths there were no explanations. One stillborn neonate was small for gestational age and the other stillborns had birth weights within normal range. The neonatal death was due to hypoxic ischaemic encephalopathy in a large for gestational age neonate. The number needed to treat with induction of labour at 41 weeks to prevent one perinatal death was 230 .

A low Apgar score ( $<7$ at five minutes) was the main contributor to the primary outcome: $1.3 \%(18 / 1381)$ in the induction group compared with $1.2 \%(16 / 1374)$ in the expectant management group (relative risk 1.12, 95\% confidence interval 0.57 to $2.19 ; \mathrm{P}=0.88$ ).

The post hoc sensitivity analysis for the primary outcome with adjustment for the minimisation variables centre and parity showed similar results (1.05, 0.65 to $1.59 ; \mathrm{P}=0.85)$.

\section{Secondary neonatal outcomes}

Table 3 shows the secondary neonatal outcomes. An Apgar score of less than 4 at five minutes occurred in $0.2 \%(3 / 1381)$ in the induction group and $0.1 \%$ $(1 / 1374)$ in the expectant management group (relative risk 2.98, 0.31 to 28.66; $\mathrm{P}=0.63)$. Fewer newborns in the induction group were admitted to a neonatal intensive care unit: $4.0 \%(55 / 1381)$ in the induction group versus $6.0 \%(82 / 1374)$ in the expectant management group $(0.67,0.48$ to $0.93 ; \mathrm{P}=0.02)$. If neonates with a major birth defect $(n=10)$ were excluded (antenatally detected major birth defect was an exclusion criterion at study entry) there was no significant difference in admittance to a neonatal intensive care unit. Fewer neonates in the induction group had jaundice treated with phototherapy or exchange transfusion: $1.2 \%(16 / 1381)$ in the induction group versus $2.3 \%$ $(32 / 1374)$ in the expectant management group (relative risk $0.50,95 \%$ confidence interval 0.27 to 0.90; $\mathrm{P}=0.03)$. Fewer neonates in the induction group had macrosomia: $4.9 \%(68 / 1381)$ in the induction group versus $8.3 \%(114 / 1379)$ in the expectant management group (0.60, 0.45 to 0.80 ; $\mathrm{P}<0.001)$. Other secondary outcomes did not differ.

\section{Maternal outcomes}

Tables 2 and 4 present the secondary maternal outcomes. Use of epidural anaesthesia was higher in the induction group: $52.8 \%(729 / 1381)$ in the induction group versus $48.5 \%(669 / 1379)$ in the expectant management group (relative risk 1.09, 95\% confidence interval 1.01 to $1.17 ; \mathrm{P}=0.03)$. The median duration of labour was shorter in the induction group (5.7 hours (interquartile range 2.9-10.3 hours) v 6.9 (3.8-11.5) hours in the expectant management group; $\mathrm{P}<0.001)$. Mode of delivery was similar in both groups: the rate of caesarean delivery was $10.4 \%(143 / 1381)$ in the induction group and $10.7 \%(148 / 1379)$ in the expectant management group (relative risk 0.96, 95\% confidence interval 0.78 to $1.20 ; \mathrm{P}=0.79$ ). Indications for caesarean delivery did not differ between the groups.

Endometritis occurred in 1.3\% (18/1381) of women in the induction group and $0.4 \%(6 / 1379)$ in the expectant management group (relative risk 3.00, $95 \%$ confidence interval 1.19 to $7.52 ; \mathrm{P}=0.02$ ). Other secondary adverse maternal outcomes, including postpartum haemorrhage and perineal tears grades 3 and 4, were similar between the groups (table 4).

Hypertensive disorders of pregnancy after randomisation (exploratory outcome) occurred in $1.4 \%$ $(19 / 1381)$ of women in the induction group compared with $3.0 \%(42 / 1379)$ of women in the expectant management group (relative risk $0.45,95 \%$ confidence interval 0.26 to $0.77 ; \mathrm{P}=0.004$ ).

\section{Per protocol analysis}

The prespecified analysis of the per protocol population included 1333 women in the induction group and 1351 women in the expectant management group. Figure 1 shows the reasons for violation of the protocol. Baseline characteristics were similar between 


\begin{tabular}{|c|c|c|c|c|}
\hline Variables & $\begin{array}{l}\text { Induction group } \\
(n=1381)\end{array}$ & $\begin{array}{l}\text { Expectant management } \\
\text { group }(n=1379)\end{array}$ & Relative risk $(95 \% \mathrm{Cl})$ & $\begin{array}{l}\mathrm{P} \text { value; mean }(95 \% \mathrm{Cl}) \\
\text { difference between groups }\end{array}$ \\
\hline \multicolumn{5}{|l|}{ Primary composite outcome } \\
\hline Primary composite outcome & $33 / 1381(2.4)$ & $31 / 1379(2.2)$ & $1.06(0.65$ to 1.73$)$ & 0.90 \\
\hline \multicolumn{5}{|l|}{ Subcomponents of primary composite outcome } \\
\hline Perinatal/neonatal mortality (stillbirth+neonatal mortality) & $0 / 1381(0.0)$ & $6 / 1379(0.4)$ & - & 0.03 \\
\hline Stillbirth & $0 / 1381(0.0)$ & $5 / 1379(0.4)$ & - & 0.06 \\
\hline Neonatal mortality (live births with death days 0-27) & $0 / 1381(0.0)$ & $1 / 1374(0.1)$ & - & 1.00 \\
\hline Neonatal morbidity & $33 / 1381(2.4)$ & 26/1374 (1.9) & $1.27(0.76$ to 2.11$)$ & 0.43 \\
\hline \multicolumn{5}{|l|}{ Subcomponents of neonatal morbidity } \\
\hline Apgar score $<7$ at 5 minutes* & $18 / 1381(1.3)$ & $16 / 1374(1.2)$ & $1.12(0.57$ to 2.19$)$ & 0.88 \\
\hline Metabolic acidosist & $14 / 661(2.1)$ & $10 / 644(1.6)$ & $1.36(0.61$ to 3.05$)$ & 0.58 \\
\hline Hypoxic ischaemic encephalopathy grades 1-3 & $2 / 1381(0.1)$ & $3 / 1374(0.2)$ & $0.66(0.11$ to 3.96$)$ & 1.00 \\
\hline Intracranial haemorrhage & $1 / 1381(0.1)$ & $2 / 1374(0.1)$ & $0.50(0.05$ to 5.48$)$ & 1.00 \\
\hline Neonatal convulsions & $1 / 1381(0.1)$ & $3 / 1374(0.2)$ & $0.33(0.03$ to 3.18$)$ & 0.62 \\
\hline Meconium aspiration syndrome & $2 / 1381(0.1)$ & $3 / 1374(0.2)$ & $0.66(0.11$ to 3.96$)$ & 1.00 \\
\hline Mechanical ventilation within first 72 hours & $3 / 1381(0.2)$ & $5 / 1374(0.4)$ & $0.60(0.14$ to 2.49$)$ & 0.72 \\
\hline Obstetric brachial plexus injury & $4 / 1381(0.3)$ & $1 / 1374(0.1)$ & $3.98(0.45$ to 35.56$)$ & 0.38 \\
\hline \multicolumn{5}{|l|}{ Additional secondary neonatal outcome variables } \\
\hline Admittance to NICU & $55 / 1381(4.0)$ & $82 / 1374(6.0)$ & $0.67(0.48$ to 0.93$)$ & 0.02 \\
\hline Apgar score $<4$ at 5 minutes* & $3 / 1381(0.2)$ & $1 / 1374(0.1)$ & $2.98(0.31$ to 28.66$)$ & 0.63 \\
\hline Therapeutic hypothermia & $1 / 1381(0.1)$ & $2 / 1374(0.1)$ & $0.50(0.05$ to 5.48$)$ & 1.00 \\
\hline Macrosomia ( $\geq 4500 \mathrm{~g})$ & $68 / 1381(4.9)$ & $114 / 1379(8.3)$ & $0.60(0.45$ to 0.80$)$ & $<0.001$ \\
\hline Birth weight (g): & $n=1381$ & $\mathrm{n}=1379$ & & \\
\hline Mean (SD) & $3815(409)$ & $3875(436)$ & - & $<0.001 ;-60.1(-91.8$ to -29.6$)$ \\
\hline Median (interquartile range) & $3804(3536-4090)$ & $3865(3570-4160)$ & - & - \\
\hline Jaundice requiring phototherapy or exchange transfusion & $16 / 1381(1.2)$ & $32 / 1374(2.3)$ & $0.50(0.27$ to 0.90$)$ & 0.03 \\
\hline Pneumonia & $8 / 1381(0.6)$ & $13 / 1374(0.9)$ & $0.61(0.25$ to 1.47$)$ & 0.38 \\
\hline Sepsis & $9 / 1381(0.7)$ & 20/1374 (1.5) & $0.45(0.20$ to 0.98$)$ & 0.06 \\
\hline \multicolumn{5}{|l|}{ Exploratory neonatal outcome variables } \\
\hline Days in NICU: & $\mathrm{n}=55$ & $\mathrm{n}=81 \neq$ & & \\
\hline Mean (SD) & $3.38(2.97)$ & $4.59(5.64)$ & - & $0.15 ;-1.21(-2.78$ to 0.20$)$ \\
\hline Median (interquartile range) & $2(1-6)$ & $3(1-6)$ & - & - \\
\hline Admittance to NICU >4 days & $34 / 55(61.8)$ & $45 / 81(55.6)$ & $1.11(0.84$ to 1.48$)$ & 0.58 \\
\hline Hypoglycaemia§ & 22/1381 (1.6) & 20/1374 (1.5) & $1.09(0.60$ to 2.00$)$ & 0.89 \\
\hline Birth traumaף & $0 / 1381(0.0)$ & $1 / 1374(0.1)$ & & 1.00 \\
\hline Small for gestational age ${ }^{\star \star}$ & 9/1381 (0.7) & $22 / 1379(1.6)$ & $0.41(0.19$ to 0.88$)$ & 0.03 \\
\hline Large for gestational age ${ }^{\star \star}$ & $21 / 1381(1.5)$ & 26/1379 (1.9) & $0.81(0.46$ to 1.43$)$ & 0.55 \\
\hline Any major birth defectt† & $14 / 1381(1.0)$ & $17 / 1379(1.2)$ & $0.82(0.41$ to 1.66$)$ & 0.72 \\
\hline Girl & $600 / 1381(43.4)$ & $623 / 1379(45.2)$ & $0.96(0.88$ to 1.05$)$ & 0.38 \\
\hline 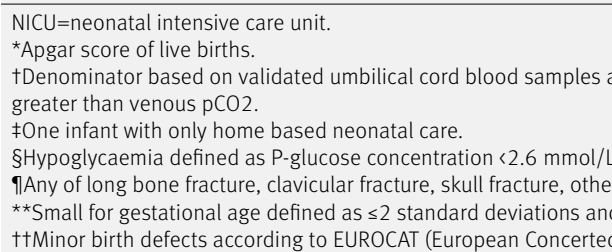 & $\begin{array}{l}\text { birth. Validated samples } \\
\text { ffter three hours. } \\
\text { neurological injury, retina } \\
\text { large for gestational age }\end{array}$ & efined as arterial $\mathrm{pH}$ less than $\mathrm{v}$ & ous pH and arterial partial pr & ssure of carbon dioxide (pCO2) \\
\hline
\end{tabular}

the groups (supplementary table $\mathrm{C}$ ). The primary perinatal adverse outcome occurred in 31 pregnancies in the induction group and 31 in the expectant management group (relative risk 1.01, 95\% confidence interval 0.62 to $1.66 ; \mathrm{P}=1.0$ ) (supplementary table $\mathrm{E}$ ). No stillbirths or neonatal deaths (0-27 days) occurred in the induction group (mortality rate $0.0 \%$ ), whereas there were five stillbirths and one neonatal death (mortality rate $0.4 \%$ ) in the expectant management group $(\mathrm{P}=0.03)$.

Supplementary tables D to F show the secondary neonatal and maternal outcomes.

\section{Subgroup analyses}

Prespecified subgroup analyses on the primary outcome and selected secondary outcomes according to parity (parity $1 v$ parity $>1$ ), maternal age ( $<35$ years $v \geq 35$ years), and body mass index (BMI) $(<30 v \geq 30)$ were performed on the intention to treat population. In the intention to treat population, analyses of the primary outcome showed no significant difference in the treatment effect according to parity, age, or BMI $(\mathrm{P}=0.29, \mathrm{P}=0.70, \mathrm{P}=0.51$, respectively, for the interaction). In total, five stillbirths and one early neonatal death occurred, all in the expectant management group; in $0.8 \%(6 / 753)$ of the nulliparous women versus $0 \%(0 / 626)$ in parous women, $1.1 \%$ $(3 / 279)$ in women aged 35 or older versus $0.3 \%$ $(3 / 1100)$ in women younger than 35 , and $1.1 \%$ (2/184) in women with a BMI of 30 or higher versus $0.4 \%(4 / 1081)$ in women with a BMI less than 30 . Because of the low mortality rate $(n=6)$ no interaction 


\begin{tabular}{|c|c|c|c|c|}
\hline Variables & $\begin{array}{l}\text { Induction group } \\
(n=1381)\end{array}$ & $\begin{array}{l}\text { Expectant management } \\
\text { group }(n=1379)\end{array}$ & $\begin{array}{l}\text { Relative risk } \\
(95 \% \mathrm{Cl})\end{array}$ & $P$ value \\
\hline \multicolumn{5}{|l|}{ Secondary maternal outcomes } \\
\hline Chorioamnionitis & $2(0.1)$ & $6(0.4)$ & $0.33(0.07$ to 1.65$)$ & 0.29 \\
\hline Shoulder dystocia & $6(0.4)$ & $4(0.3)$ & $1.50(0.42$ to 5.30$)$ & 0.76 \\
\hline Perineal tear grade 3 or 4 & $40(2.9)$ & $50(3.6)$ & $0.80(0.53$ to 1.20$)$ & 0.33 \\
\hline Postpartum haemorrhage (>1000 mL) & $140(10.1)$ & $146(10.6)$ & $0.96(0.77$ to 1.19$)$ & 0.75 \\
\hline Wound infection & $4(0.3)$ & $3(0.2)$ & $1.33(0.30$ to 5.94$)$ & 1.00 \\
\hline Urinary tract infection, including pyelonephritis & $5(0.4)$ & $7(0.5)$ & $0.71(0.23$ to 2.24$)$ & 0.77 \\
\hline Endometritis & $18(1.3)$ & $6(0.4)$ & 3.00 (1.19 to 7.52$)$ & 0.02 \\
\hline Sepsis & $0(0.0)$ & $0(0.0)$ & - & 1.00 \\
\hline \multicolumn{5}{|l|}{ Exploratory maternal outcomes } \\
\hline Pre-eclampsia, gestational hypertension, eclampsia & $19(1.4)$ & $42(3.0)$ & $0.45(0.26$ to 0.77$)$ & 0.004 \\
\hline Uterine rupture & $0(0.0)$ & $0(0.0)$ & - & 1.00 \\
\hline Cervical tear & $5(0.4)$ & $8(0.6)$ & $0.62(0.20$ to 1.90$)$ & 0.58 \\
\hline Venous thromboembolism & $0(0.0)$ & $1(0.1)$ & - & 1.00 \\
\hline Maternal admission to intensive care unit & $2(0.1)$ & $0(0.0)$ & - & 0.50 \\
\hline Maternal death & $0(0.0)$ & $0(0.0)$ & - & 1.00 \\
\hline
\end{tabular}

analysis on mortality could be performed. Among nulliparous women, the rate of caesarean delivery was $16.7 \%(127 / 762)$ in the induction group and $17.3 \%(130 / 753)$ in the expectant management group $(\mathrm{P}=0.81)$.

When testing if the effect of induction versus expectant management was similar across centres (Stockholm centres versus other centres-that is, offering or not offering a routine ultrasound scan at 41 weeks) no significant interaction effect was found for the primary outcome $(\mathrm{P}=0.19)$ in the intention to treat population. Perinatal mortality in the expectant management group was $0.0 \%(0 / 557)$ in Stockholm centres versus $0.7 \%(6 / 822)$ in the other centres.

\section{Discussion}

In this large randomised trial, comparing induction of labour at 41 weeks with expectant management and induction at 42 weeks, we found no significant difference in the primary composite adverse perinatal outcome $-2.4 \%$ in the induction group and $2.2 \%$ in the expectant management group (relative risk 1.06, 95\% confidence interval 0.65 to $1.73, \mathrm{P}=0.90$ ). Perinatal mortality was, however, significantly lower in the induction group (no deaths) than expectant management group (five intrauterine deaths, one neonatal death; $\mathrm{P}=0.03)$. Furthermore, the induction group had lower admittance to a neonatal intensive care unit, fewer infants with neonatal jaundice requiring therapy, and fewer macrosomic infants. We found no significant difference in caesarean delivery rates between groups.

\section{Comparison with previous studies}

Post-term pregnancy ( $\geq 42$ weeks) is associated with an increased risk of adverse perinatal morbidity and mortality. ${ }^{3-5}$ The risk appears to increase gradually after 40 weeks. $^{3}$ w $^{13}$ Results from most metaanalyses indicate that a policy of induction before 42 full weeks is associated with decreased perinatal mortality. ${ }^{92-24}$
In our study all perinatal deaths occurred in nulliparous women. Nulliparity is notalways recognised as a factor conferring increased risk of perinatal mortality, 2526 but our results agree with a Swedish register study where stillbirths were significantly more common in nulliparous than multiparous women and the increase in neonatal mortality was seen at 41 completed weeks in nulliparous women but not until 42 weeks in multiparous women. ${ }^{3}$ If this finding can be replicated in future studies, it could mean that nulliparous women may require particular attention, and interventions such as labour induction might be even more important in this group.

The benefit of early induction is supported by a recently published open label multicentre randomised trial (INDEX) from the Netherlands including 1801 women, in which induction at 41 weeks was associated with a lower composite adverse perinatal outcome (1.7\%) compared with expectant management until 42 weeks $(3.1 \%$; $\mathrm{P}=0.045) .{ }^{12}$ The perinatal mortality rate did not, however, differ significantly between the groups, with one death in the 41 weeks group and two in the 42 weeks group.

It could be argued that the higher mortality in the expectant management group in our study is partly due to lack of routine fetal surveillance with cardiotocography or ultrasonography between 41 and 42 weeks unless there were clinical signs of complications. In general, however, the adverse perinatal outcomes were not higher in the expectant management group in our trial compared with the INDEX trial, and the median gestational age at delivery was higher in the expectant management group in our trial (292 days) than in the INDEX trial (289 days), which could augment mortality rates. No perinatal deaths occurred among women recruited in the Stockholm region, where all women are offered a routine ultrasound scan at 41 weeks (before randomisation), with the aim of identifying women with an increased risk for adverse outcomes. However, the rarity of perinatal death limits the power 
of a subanalysis by centre. Furthermore, two of the five cases of hypoxic ischaemic encephalopathy occurred in Stockholm and the composite neonatal morbidity was similar between Stockholm $(24 / 1122=2.1 \%)$ and the other centres $(35 / 1633=2.1 \%)$, which does not support that the 41 week ultrasound scan was critical. It is also uncertain to what extent ultrasonography or cardiotocography usually performed at two or three day intervals can prevent intrauterine or neonatal deaths, ${ }^{72627}$ and the evidence supporting that fetal monitoring prevents complications of post-maturity is considered weak. $^{7}$

The occurrence of endometritis was significantly higher in the induction group than expectant management group, which was unexpected but might well be a chance finding. Recent studies indicate that infectious morbidity is not higher for mechanical methods than for drugs for cervical dilation, ${ }^{28}$ and the occurrence of endometritis is similar or lower in our trial than reported in most studies on labour induction. ${ }^{28-30}$ Furthermore, the frequency of other maternal infections (chorioamnionitis, wound infections, urinary tract infections) and neonatal infections (sepsis, pneumonia) was not higher in the induction group.

\section{Strengths and weaknesses of this study}

We carried out a large national multicentre randomised controlled trial comparing induction at 41 weeks with expectant management and induction at 42 weeks, the latter being standard of care in Sweden at present. Regardless that only a minority of eligible women were informed or accepted participation (fig 1), the study population was representative of a Swedish low risk population according to most baseline characteristics (supplementary table B). Another strength is that the participants were managed at the same level of care and methods of induction were applied irrespective of allocation arm, which was not always the case in previous randomised trials on post-term pregnancies. ${ }^{12} 31$

Our trial does have some limitations. Although it could seem contradictory that a significant difference was found between groups in perinatal mortality, we found no difference in the composite adverse neonatal outcome. However, five of the six deaths were stillbirths in our trial, which have a quite different cause and array of risk factors ${ }^{32}$ compared with neonatal mortality and morbidity. ${ }^{33}$ Placental abnormality or dysfunction, umbilical cord complications, and growth restriction are considered causes of stillbirth ${ }^{2} 32$ that could well be of increasing importance in late and post-term pregnancies.

Another problem is that the composite primary outcome was defined somewhat broadly, predominated by an Apgar score of less than 7 at five minutes, which according to recent data might be a relatively weak predictor of more serious outcomes such as neurological morbidity and mortality, therefore an Apgar of less than 4 at five minutes is probably preferable. ${ }^{34}$ The advantage of composite outcomes, however, is that the number of cases in each arm can be reduced, and carrying out the study becomes more realistic.

Pregnant women were not involved in the design of our trial, which is a limitation ${ }^{35}$ despite our impression that management of late term and post-term pregnancies is a prioritised area of research for many women. In a separate survey, to be published, we will be addressing pregnant women's experiences in the 41 and 42 week groups.

The fact that half of the women (those recruited in the Stockholm region) underwent ultrasound measurement of amniotic fluid volume and abdominal diameter at 41 weeks, whereas such examinations were not performed systematically at the other centres might be regarded as both a limitation and a strength. It is difficult to determine whether outcomes were affected by this difference in policy, whereas such a management increases generalisability and reflects current obstetric practice in Sweden. ${ }^{36}$

It is not clear whether the results are broadly generalisable. The study did include university, regional, and local hospitals, and women from 17 countries were eligible for inclusion. Different methods for labour induction, according to local practice, were allowed, and one large region used an extra ultrasound scan in gestational week 41 before inclusion. All these strategies increase the generalisability of the results.

Although we performed several significance tests, also for secondary and exploratory outcomes, we have not corrected for multiple comparisons owing to the risk of not finding differences of high clinical importance for women.

\section{Conclusions and policy implications}

Our study found that induction of labour at 41 weeks compared with expectant management and induction at 42 weeks does not alter the composite perinatal outcome, the primary outcome of this study. However, a reduction of the secondary outcome perinatal mortality is observed without increasing adverse maternal outcome. The number needed to treat with induction of labour at 41 weeks to prevent one perinatal death was 230 , which is lower than previous estimates. ${ }^{92223}$ Although these results should be interpreted cautiously, based on previous reports and the results of the present trial we suggest that labour induction should be offered to women at 41 weeks+0 days ${ }^{12}$ or earlier ${ }^{1137}$ and could be one (of few) interventions that reduces the rate of stillbirths.

\section{AUTHOR AFFILIATIONS}

${ }^{1}$ Department of Obstetrics and Gynaecology, Institute of

Clinical Sciences, Sahlgrenska Academy, Gothenburg

University, Sahlgrenska University Hospital, 41685 Gothenburg, Sweden

${ }^{2}$ Department of Women's and Children's Health, Karolinska Institutet, Karolinska University Hospital, Stockholm, Sweden ${ }^{3}$ Institute of Health and Care Sciences, Sahlgrenska Academy, Gothenburg University, Sweden

${ }^{4}$ Department of Clinical Sciences, Karolinska Institutet, Danderyd Hospital, Stockholm, Sweden

${ }^{5}$ Department of Obstetrics and Gynaecology, Faculty of Medicine and Health, Örebro University, Örebro, Sweden 
${ }^{6}$ Department of Women's and Children's Health, Uppsala University, Uppsala, Sweden

${ }^{7}$ Center for Clinical Research Dalarna, Uppsala University, Sweden

${ }^{8}$ Department of Paediatrics, Institute of Clinical Sciences, Sahlgrenska Academy, Gothenburg University, Sahlgrenska University Hospital, Gothenburg, Sweden

${ }^{9}$ Department of Medicine, Solna, Clinical Epidemiology Division, Karolinska Institutet, Stockholm, Sweden

Jonas Eriksson Söderling provided data from the Swedish Pregnancy Register and performed the statistical analysis for the Data Safety Monitoring Board reports, Stellan Håkansson provided data from the Swedish Neonatal Quality Register, Jesper Brodin provided data from Statistics Sweden, and Agneta Cedefors-Blom helped with secretarial assistance. Mattias Molin and Per Ekman, the Statistical Consulting Group, Gothenburg, performed the statistical analyses. Therese Svanberg at the Medical Library at Sahlgrenska University Hospital performed the literature search. Thanks to the members of the Data Safety Monitoring Board, Hans Wedel (chairman), Lars-Åke Mattson, and Elisabeth Jangsten for their assistance and to the women who participated in the trial.

The SWEPIS study group: the midwives and doctors responsible at the local centres were: Uppsala University Hospital: Irina Sylwe; South Älvsborg Hospital: Lena Loubelo, Carolina Bergerum, and Serney Böö; Department of Gynaecology Närhälsan, Mölndal: Maria Bullarbo; Sahlgrenska University Hospital, Göteborg: PhD candidates Anna Wessberg and Helena Nilver, and Pia Hempel, Martina Söderlund, Erica Ginström Ernstad, and Monica Eriksson Orrskog; Stockholm: Karolinska University Hospital Huddinge and Solna, South Hospital, Danderyd Hospital, South BB, Södertälje Hospital: Helen Fagraeus, Annelie Sjölund, and Eva Itzel Wiberg; Halland Hospital: Elisabeth Johansson, Sandra Holmström, Åsa Ponten, and Maud Ankardal; Örebro Hospital: Inger Nydahl, Sofia Saarväli, and Camilla Hartin; Falun Hospital: Elisabeth Nordström and Kerstin Fransson; Visby Hospital: Madelen Jacobsson; and North Älvsborg Hospital: Maria Olsson and Anna Hagman.

Contributors: UBW and SS are joint first authors and contributed equally to the study. UBW, HH, VS, and HE conceived and designed the study. UBW, HH, AW, SS, AKW, MJ, HF, and JW oversaw recruitment of study participants and collection of data at the local centres. UBW, $\mathrm{HH}, \mathrm{CB}, \mathrm{HE}, \mathrm{OS}$, and SS wrote the statistical analysis plan together with two statisticians (Mattias Molin and Nils-Gunnar Pehrsson, the Statistical Consulting Group, Gothenburg). UBW and MA did the data cleaning together with statistician Mattias Molin and Per Ekman. UBW, HH, CB, SS, MA, LL, VS, SBW, OS, GW, HE, and AW interpreted the data. UBW, MA, AW, SS, and $\mathrm{HH}$ wrote the first draft of the manuscript, which was then critically reviewed and revised by the other coauthors. $\mathrm{HE}, \mathrm{OS}$, and $\mathrm{HH}$ are joint senior authors. All authors approved the final version of the manuscript for submission. UBW, SS, HE, OS, and $\mathrm{HH}$ are guarantors. All authors had full access to all the data in the study and take responsibility for the integrity of the data and the accuracy of the data analysis. The corresponding author attests that all listed authors meet authorship and that no others meeting the criteria have been omitted

Funding: This study was supported by the Swedish state under the agreement between the Swedish government and the county councils, the ALF-agreement (ALFGBG-440301, ALFGBG-718721, ALFGBG-70940, ALFGBG-426401), the Health Technology Centre at Sahlgrenska University Hospital, the Foundation of the Health and Medical care committee of the Region of Vastra Gotaland, Sweden (VGFOUREG387351, VGFOUREG640891, VGFOUREG854081), Hialmar Svensson Foundation, the foundation Mary von Sydow, born Wijk donation fund, Uppsala-Örebro regional research council (RFR556711, RFR-736891), Region Örebro County research committee (OLL-715501), the ALF-agreement in Stockholm (ALF-561222. ALF-562222, ALF-563222), and Centre for Clinical Research DalarnaUppsala University, Sweden (CKFUU-417011). The funders had no role in study design, data collection, data analysis, data interpretation, or writing of the report. The researchers were independent of the funders.

Competing interests: All authors have completed the ICMJE uniform disclosure form at www.icmje.org/coi disclosure.pdf and declare: no support from any organisation for the submitted work; no financial relationship with any organisation that might have an interest in the submitted work in the previous three years; no other relationships or activities that could appear to have influenced the submitted work. AKW has received free reagents (PIGF) from Roche for a prediction study of pre-eclampsia.
Ethical approval: This study was approved by the regional ethics board in Gothenburg in May 2014 (Dnr: 285-14) and later its complementary applications (T 905-15, T 291-16, T 1180-16, T 330-17, T 1066-17, T 087-18, T 347-18, T 961-18, T 1110-18). All participants gave informed written consent before taking part in the study.

Data sharing: The full dataset is available from the corresponding author on reasonable request.

The corresponding author (UBW) affirms that this manuscript is an honest, accurate, and transparent account of the study being reported; that no important aspects of the study have been omitted; and that any discrepancies from the study as planned have been explained. The corresponding author (UBW) had the final responsibility for the decision to submit for publication.

This is an Open Access article distributed in accordance with the Creative Commons Attribution Non Commercial (CC BY-NC 4.0) license, which permits others to distribute, remix, adapt, build upon this work non-commercially, and license their derivative works on different terms, provided the original work is properly cited and the use is noncommercial. See: http://creativecommons.org/licenses/by-nc/4.0/.

1 Olesen AW, Westergaard JG, Olsen J. Perinatal and maternal complications related to postterm delivery: a national registerbased study, 1978-1993. Am J Obstet Gynecol 2003;189:222-7. doi:10.1067/mob.2003.446

2 Lawn JE, Blencowe H, Waiswa P, et al, Lancet Ending Preventable Stillbirths Series study group, Lancet Stillbirth Epidemiology investigator group. Stillbirths: rates, risk factors, and acceleration towards 2030. Lancet 2016;387:587-603. doi:10.1016/S01406736(15)00837-5

3 Ingemarsson I, Källén K. Stillbirths and rate of neonatal deaths in 76,761 postterm pregnancies in Sweden, 1982-1991: a register study. Acta Obstet Gynecol Scand 1997;76:658-62. doi:10.3109/00016349709024606

4 Nakling J, Backe B. Pregnancy risk increases from 41 weeks of gestation. Acta Obstet Gynecol Scand 2006;85:663-8. doi:10.1080/00016340500543733

5 Smith GC. Life-table analysis of the risk of perinatal death at term and post term in singleton pregnancies. Am J Obstet Gynecol 2001:184:489-96. doi:10.1067/mob.2001.109735

6 WHO recommendations: induction of labour at or beyond term. (accessed June 9 2019). https://apps.who.int/iris/bitstream/hand le/10665/277233/9789241550413-eng.pdf

7 American College of Obstetricians and Gynecologists. Practice bulletin no. 146: Management of late-term and postterm pregnancies. Obstet Gynecol 2014;124:390-6. doi:10.1097/01. AOG.0000452744.06088.48

8 Induction of labour: new NICE quality standard. Midwives 2014;17:8.

9 Middleton P, Shepherd E, Crowther CA. Induction of labour for improving birth outcomes for women at or beyond term. Cochrane Database Syst Rev 2018;5:CD004945. doi:10.1002/14651858. CD004945.pub4

10 Elden $\mathrm{H}$, Hagberg $\mathrm{H}$, Wessberg A, et al. Study protocol of SWEPIS a Swedish multicentre register based randomised controlled trial to compare induction of labour at 41 completed gestational weeks versus expectant management and induction at 42 completed gestational weeks. BMC Pregnancy Childbirth 2016;16:49. doi:10.1186/s12884-016-0836-9

11 Grobman WA, Rice MM, Reddy UM, et al, Eunice Kennedy Shriver National Institute of Child Health and Human Development Maternal-Fetal Medicine Units Network. Labor Induction versus Expectant Management in Low-Risk Nulliparous Women. N Engl J Med 2018;379:513-23. doi:10.1056/NEJMoa1800566

12 Keulen JK, Bruinsma A, Kortekaas JC, et al. Induction of labour at 41 weeks versus expectant management until 42 weeks (INDEX): multicentre, randomised non-inferiority trial. BMJ 2019;364:1344 doi:10.1136/bmj.1344

13 Muglu J, Rather H, Arroyo-Manzano D, et al. Risks of stillbirth and neonatal death with advancing gestation at term: A systematic review and meta-analysis of cohort studies of 15 million pregnancies. PLoS Med 2019:16:e1002838 doi:10.1371/journal.pmed.1002838

14 Gelisen O, Caliskan E, Dilbaz S, et al. Induction of labor with three different techniques at 41 weeks of gestation or spontaneous follow-up until 42 weeks in women with definitely unfavorable cervical scores. Eur J Obstet Gynecol Reprod Biol 2005;120:164-9. doi:10.1016/j.ejogrb.2004.08.013

15 Bruinsma A, Keulen J, Bakker J, et al. Induction of labor at 41 weeks or expectant managment until 42 weeks- preliminary results of the INDEX trial. Am J Obstet Gynecol 2017;216 (1 Suppl):S27-S28 (abstr no 40).

16 Stephansson O, Petersson K, Björk C, Conner P, Wikström AK. The Swedish Pregnancy Register - for quality of care improvement 
and research. Acta Obstet Gynecol Scand 2018;97:466-76. doi:10.1111/aogs.13266

17 Marsál K, Persson PH, Larsen T, Lilja H, Selbing A, Sultan B. Intrauterine growth curves based on ultrasonically estimated foetal weights. Acta Paediatr 1996;85:843-8. doi:10.1111/j.1651-2227.1996.tb14164.x

18 Norman M, Källén K, Wahlström E, Håkansson S, SNQ Collaboration. The Swedish Neonatal Quality Register - contents, completeness and validity. Acta Paediatr 2019;108:1411-8. doi:10.1111/apa.14823

19 Ludvigsson JF, Otterblad-Olausson P, Pettersson BU, Ekbom A The Swedish personal identity number: possibilities and pitfalls in healthcare and medical research. Eur J Epidemiol 2009;24:659-67. doi:10.1007/s10654-009-9350-y

20 Göransson M, Magnusson A, Heilig M. Identifying hazardous alcoho consumption during pregnancy: implementing a research-based model in real life. Acta Obstet Gynecol Scand 2006;85:657-62. doi:10.1080/00016340600589677

21 European Surveillance of Congenital Anomalies (European Concerted Action on Congenital Anomalies and Twins (EUROCAT). Data collection. Guideline for Registration. (accessed May 9, 2019). www. eurocat-network.eu/content/EUROCAT-Guide-1.4-section-3.2.

22 Gülmezoglu AM, Crowther CA, Middleton P, Heatley E. Induction of labour for improving birth outcomes for women at or beyond term. Cochrane Database Syst Rev 2012;6:CD004945. doi:10.1002/14651858.CD004945.pub3

23 Hussain AA, Yakoob MY, Imdad A, Bhutta ZA. Elective induction fo pregnancies at or beyond 41 weeks of gestation and its impact on stillbirths: a systematic review with meta-analysis. BMC Public Health 2011:11(Suppl 3):S5. doi:10.1186/1471-2458-11-S3-S5

24 Wennerholm UB, Hagberg H, Brorsson B, Bergh C. Induction of labor versus expectant management for post-date pregnancy: is there sufficient evidence for a change in clinical practice?Acta Obstet Gynecol Scand 2009;88:6-17. doi:10.1080/00016340802555948

25 Lawn JE, Wilczynska-Ketende K, Cousens SN. Estimating the causes of 4 million neonatal deaths in the year 2000. Int J Epidemiol 2006;35:706-18. doi:10.1093/ije/dyl043

26 Delaney M, Roggensack A, CLINICAL PRACTICE OBSTETRICS COMMITTEE. Guidelines for the management of pregnancy at $41+0$ to 42+0 weeks. J Obstet Gynaecol Can 2008;30:800-10. doi:10.1016/ S1701-2163(16)32945-0

27 Nabhan AF, Abdelmoula YA. Amniotic fluid index versus single deepest vertical pocket as a screening test for preventing adverse pregnancy outcome. Cochrane Database Syst Rev 2008;3:CD006593. doi:10.1002/14651858.CD006593.pub2
28 McMaster K, Sanchez-Ramos L, Kaunitz AM. Evaluation of a Transcervical Foley Catheter as a Source of Infection: A Systematic Review and Meta-analysis. Obstet Gynecol 2015;126:539-51. doi:10.1097/AOG.0000000000001002

29 Heinemann J, Gillen G, Sanchez-Ramos L, Kaunitz AM. Do mechanical methods of cervical ripening increase infectious morbidity? A systematic review. Am J Obstet Gynecol 2008;199:177-87, discussion 187-8. doi:10.1016/j.ajog.2008.05.005

30 Gommers ISM, Diederen M, Wilkinson C, Turnbull D, Mol BW]. Risk of maternal, fetal and neonatal complications associated with the use of the transcervical balloon catheter in induction of labour: A systematic review. Eur J Obstet Gynecol Reprod Biol 2017;218:7384. doi:10.1016/j.ejogrb.2017.09.014

31 Hannah ME, Hannah WJ, Hellmann J, Hewson S, Milner R, Willan A, The Canadian Multicenter Post-term Pregnancy Trial Group. Induction of labor as compared with serial antenatal monitoring in post-term pregnancy. A randomized controlled trial. N Engl J Med 1992;326:1587-92. doi:10.1056/ NEJM199206113262402

32 Flenady V, Middleton P, Smith GC, et al, Lancet's Stillbirths Series steering committee. Stillbirths: the way forward in high-income countries. Lancet 2011;377:1703-17. doi:10.1016/S01406736(11)60064-0

33 Blencowe H, Calvert C, Lawn JE, Cousens S, Campbell OM. Measuring maternal, foetal and neonatal mortality: Challenges and solutions. Best Pract Res Clin Obstet Gynaecol 2016;36:14-29. doi:10.1016/j. bpobgyn.2016.05.006

34 Committee Opinion No. 644: The Apgar Score. Obstet Gynecol 2015 126: e52-5.35

35 Cheyne H, McCourt C, Semple K. Mother knows best: developing a consumer led, evidence informed, research agenda for maternity care. Midwifery 2013;29:705-12. doi:10.1016/j. midw.2012.06.015

36 Grunewald C, Håkansson S, Saltvedt S, Källén K. Significant effects on neonatal morbidity and mortality after regional change in management of post-term pregnancy. Acta Obstet Gynecol Scand 2011;90:26-32. doi:10.1111/j.1600-0412.2010.01019.x

37 Walker KF, Bugg GJ, Macpherson M, et al, 35/39 Trial Group. Randomized Trial of Labor Induction in Women 35 Years of Age or Older. N Engl J Med 2016;374:813-22. doi:10.1056/ NEJMoa1509117

Supplementary information: tables A to F 